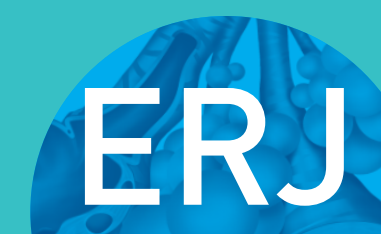

open research
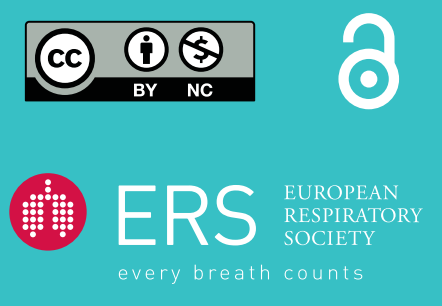

\section{Intake of multivitamin supplements and incident asthma in Norwegian adults: the HUNT study}

\author{
Lin Jiang ${ }^{1}$, Ben Brumpton ${ }^{1,2}$, Arnulf Langhammer ${ }^{1}$, Yue Chen $^{3}$ and \\ Xiao-Mei Mai ${ }^{1}$
}

Affiliations: ${ }^{1}$ Dept of Public Health and General Practice, Faculty of Medicine, Norwegian University of Science and Technology, Trondheim, Norway. ${ }^{2}$ Liason Committee between the Central Norway Regional Health Authority and the Norwegian University of Science and Technology, Trondheim, Norway. ${ }^{3}$ School of Epidemiology, Public Health and Preventive Medicine, Faculty of Medicine, University of Ottawa, Ottawa, Canada.

Correspondence: Xiao-Mei Mai, Dept of Public Health and General Practice, Norwegian University of Science and Technology (NTNU), Postbox 8905, MTFS, N0-7491 Trondheim, Norway. E-mail: xiao-mei.maiantnu.no

ABSTRACT Although intake of multivitamin supplements is becoming increasingly popular, the relationship between intake of multivitamin supplements and incident asthma remains unclear. Prospective studies in adults with long-term follow-up are especially scarce. Our objective was to investigate the association between intake of multivitamin supplements and asthma development in Norwegian adults.

We followed 16952 adult subjects from the second survey of the Nord-Trøndelag Health Study (1995-1997) up to 2006-2008, who, at baseline, were free of asthma and provided information on their intake of multivitamin supplements and cod liver oil. Regular intake of multivitamin supplements or cod liver oil was defined as daily intake for $\geqslant 3$ months during the year prior to baseline. Incident asthma was defined as reported new-onset asthma after the 11-year follow-up.

Intake of multivitamin supplements only was associated with an increased odds ratio for incident asthma (OR 1.55, 95\% CI 1.12-2.13) after adjustment for a number of common confounding factors (model I). Similar odds ratios were found for intake of cod liver oil only and for intake of both supplements (1.59 and 1.73, respectively).

Regular intake of multivitamin supplements was associated with an increased odds ratio for incident asthma in Norwegian adults.

@ERSpublications

Multivitamin supplement intake was associated with an increased odds ratio for incident asthma in Norwegian adults http://ow.ly/RNBxo

This article has supplementary material available from openres.ersjournals.com

Received: June 222015 | Accepted after revision: Aug 302015

Support statement: This study was supported by funds from the Research Council of Norway (project number 201895/ V50). The Lung Study in HUNT 2 and HUNT 3 received funding from AstraZeneca Norway. B. Brumpton received a post-doctoral fellowship grant from the Liaison Committee between the Central Norway Regional Health Authority and the Norwegian University of Science and Technology. None of the funding sources was involved in any aspect of the study design, conduct, analysis, interpretation of data, or writing the report. Funding information for this article has been deposited with FundRef.

Conflict of interest: Disclosures can be found alongside this article at openres.ersjournals.com

Copyright $\odot$ ERS 2015. This article is open access and distributed under the terms of the Creative Commons Attribution Non-Commercial Licence 4.0. 


\section{Introduction}

Intake of vitamin supplements to improve health is becoming increasingly popular [1]. Multivitamin supplements are the most commonly used forms of vitamin supplements [1]. The majority of studies, both observational studies and randomised trials, has shown no overall benefit of multivitamin supplements on cancer or cardiovascular diseases $[1,2]$. However, some studies have shown an increased risk of breast and prostate cancers in relation to multivitamin supplement use $[3,4]$. These surprising and unintuitive results have led to ongoing debate.

The relationship between multivitamin supplements use and asthma risk has been previously studied in children with inconsistent results $[5,6]$. So far, there have been no prospective studies with a long follow-up time in adults.

On the Norwegian market, brands of multivitamin supplements are limited and the vitamin components are stable chemical synthetic substances in either water-miscible or solid forms (containing mainly vitamins A, B, C, D and E) $[7,8]$. Components in the multivitamin supplements are kept within the normal ranges according to the Norwegian daily recommendation [8]. In contrast, cod liver oil, also a commonly used type of vitamin supplement in Norway [7], contained a high concentration of naturally oil-based vitamin A $(1000 \mu \mathrm{g}$ per $5 \mathrm{~mL})$ before 1999 [9]. The high concentration of vitamin A in this former formula of cod liver oil was hypothesised to be the main reason for an increased risk of asthma associated with cod liver oil intake among Norwegian adults observed in our previous study [10]. In the current analysis, we further evaluated the risk of incident asthma among adults who regularly used 1) cod liver oil only, 2) multivitamin supplements only or 3) both, compared to those who did not regularly use any of these supplements.

\section{Methods}

Study design

The Nord-Trøndelag Health Study (HUNT) is one of the largest prospective population-based studies in Norway. It consists of three surveys that were carried out separately in 1984-1986 (HUNT1), 1995-1997 (HUNT2) and 2006-2008 (HUNT3) [11]. All adults aged 19 years or older living in the county of Nord Trøndelag were invited to participate at each cycle [12]. Nord-Trøndelag is considered to be fairly representative of the Norwegian population regarding geography, economy, sources of income, age distribution, morbidity and mortality [11]. The population of the HUNT study is reasonably homogenous, with $>97 \%$ Caucasian inhabitants. The response rate was $69.5 \%(\mathrm{n}=65237)$ for HUNT2 and about 37059 adults took part in both HUNT2 and HUNT3 (figure 1) [12]. Among the 37059 participants, we selected those who were $<65$ years of age in HUNT3 $(n=25616)$ to establish our study cohort to keep a low possibility of misclassification of asthma with chronic obstructive pulmonary disease (COPD).

FIGURE 1 A flow chart of study cohort. HUNT: Nord-Trøndelag Health Study; HUNT2: 1995-1997; HUNT3: 2006-2008.

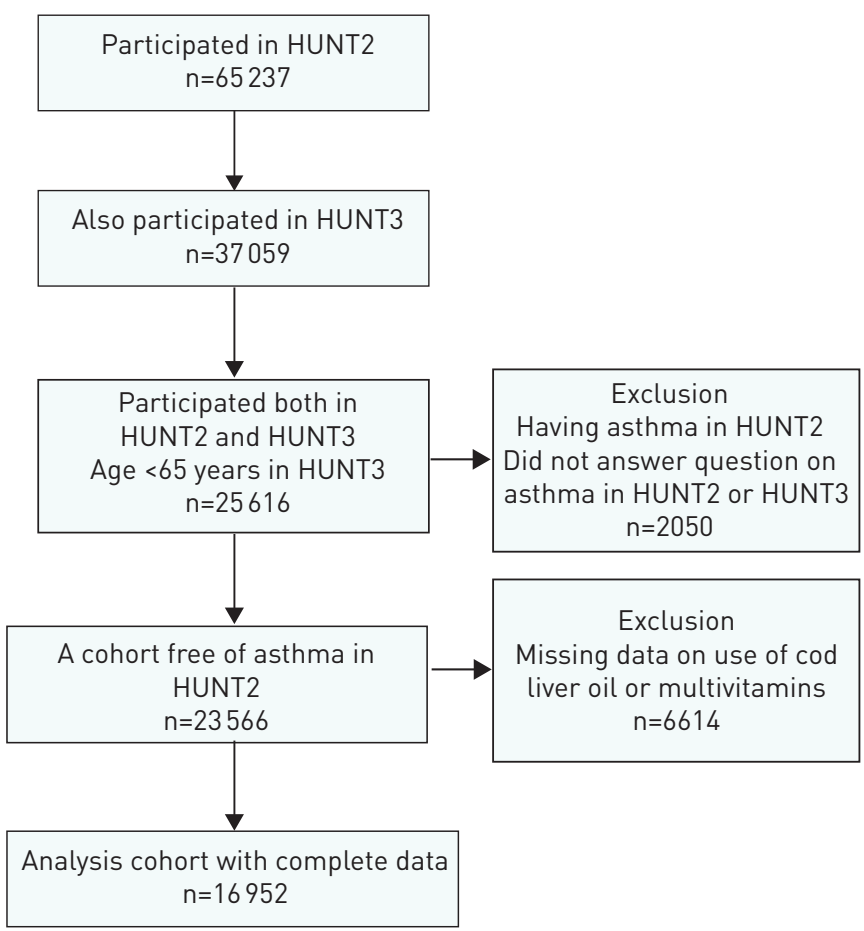




\section{Incident asthma}

The same question regarding asthma, "Do you have or have you had asthma?", was asked in both HUNT2 and HUNT3. We excluded 2050 subjects because they either reported having asthma in HUNT2 or did not answer the question on asthma in HUNT2 or HUNT3. This left 23566 subjects defined as free from asthma at baseline. Cases of incident asthma over an average 11-year follow-up were defined as participants who reported asthma for the first time in HUNT3. Furthermore, we used a stricter definition of asthma for sensitivity analyses; the participants were free from wheeze and asthma at baseline and reported asthma at follow-up in combination with use of asthma medications (e.g. inhaled corticosteroids and/or $\beta_{2}$-agonists). Participants who were free from wheeze and asthma both at baseline and at follow-up served as the reference group. Wheeze referred to attacks of wheezing or breathlessness during the last 12 months. In Norway, asthma medications are prescribed to patients after a doctor has confirmed the diagnosis.

\section{Cod liver oil use and multivitamin supplements at baseline}

Total months of daily use of cod liver oil and multivitamin supplements during the past 12 months prior to HUNT2 were sought in questionnaires. We defined daily intake of either cod liver oil or multivitamin supplements for $\geqslant 3$ months as regular intake. There were 16952 subjects with complete data on use of cod liver oil and multivitamin supplements at baseline, and 6614 with missing information on exposure to either cod liver oil, multivitamin supplements or both. The regular intake of supplements was categorised as 1) use of cod liver oil only, 2) use of multivitamin supplements only and 3) use of both. Nonusers or those with a daily intake of cod liver oil or multivitamin supplements for $<3$ months served as the reference group.

\section{Other baseline variables}

Data on all baseline variables were collected in a clinical examination or by questionnaires in HUNT2. These variables included age, sex, smoking status, average hours of light physical activity per week, years of education, social benefit receipt, economic difficulties, family history of asthma, body mass index (BMI), alcohol consumption, symptoms of anxiety and depression, and any chronic diseases. Current smokers responded "yes" to "Do you smoke cigarettes daily?". Former smokers responded "no" to "Do you smoke cigarettes daily?" and "no" to "Never smoked daily". Never smokers responded "no" to "Do you smoke cigarettes daily?" and "yes" to "Never smoked daily". Social benefit recipients were those who reported receiving any public welfare benefits. Economic difficulties referred to those who had difficulties to meet the costs of food, transport or housing. Weight and height were measured by health professionals. BMI was grouped into three categories $\left(<25.0,25.0-29.9\right.$ and $\left.\geqslant 30.0 \mathrm{~kg} \cdot \mathrm{m}^{-2}\right)$ according to the recommendations of the World Health Organization [13]. Alcohol consumption was assessed by the participant's response to the question "How many times a month do you normally drink alcohol?". Symptoms of anxiety and depression were assessed by the Hospital Anxiety and Depression Scale (HADS) [14]. It included two sets of questions about the participant's feeling in the past week, seven for symptoms of anxiety and seven for symptoms of depression, with a four-point ordinal scale of symptom severity (0-3) for each question. The total score for anxiety or depression therefore ranges from 0 to 21 [15]. "Any chronic diseases at baseline" was defined as any long-term $(\geqslant 1$ year) illness or injury of a physical or psychological nature that impaired functioning in everyday life.

\section{Statistical analysis}

Descriptive statistics for baseline characteristics of the cohort were presented in table 1 . The cumulative incidence of asthma over the approximate 11-year follow-up was compared among four groups using nonusers or those with a daily intake of cod liver oil or multivitamin supplements for $<3$ months as the reference group in logistic regression models, which yielded odds ratios and $95 \%$ confidence intervals. Model I included age (19-29, 30-39, 40-49 and 50-55 years), sex (men/women), smoking (current, former, never and unknown), light physical activity $(<1,1-2$ and $\geqslant 3 \mathrm{~h}$ per week, and unknown), education $(<10$, $10-12$ and $\geqslant 13$ years, and unknown), social benefit (yes, no and unknown), economic difficulties (yes, no and unknown), family history of asthma (yes, no and unknown) and BMI categories $(<25.0,25.0-29.9$ and $\geqslant 30.0 \mathrm{~kg} \cdot \mathrm{m}^{-2}$, and unknown) at baseline as covariates. Model II was adjusted for alcohol consumption in addition to the covariates in model I. Model III was adjusted for symptoms of anxiety and depression, and chronic disease at baseline, in addition to covariates in model I. Sensitivity analysis was performed in 14939 subjects by using the stricter definition of asthma to test the robustness of our results. All statistical analyses were performed with STATA, release v. 12.0.19 (StatCorp, College Station, TX, USA).

\section{Ethics}

The study was approved by the Regional Committee for Medical Research Ethics. All participants signed informed written consent before participating in HUNT. 
TABLE 1 Use of cod liver oil and multivitamin supplements by baseline characteristics in the Nord-Trøndelag Health Study in 1995-1997 to 2006-2008 ( $N=16952)$

\begin{tabular}{|c|c|c|c|c|}
\hline & Reference group ${ }^{\#}$ & Cod liver oil only & Multivitamin supplements only & Use of both \\
\hline Age at baseline years & $38.8 \pm 8.8$ & $41.6 \pm 8.1$ & $40.4 \pm 8.9$ & $41.9 \pm 8.6$ \\
\hline \multicolumn{5}{|l|}{ HADS } \\
\hline Anxiety & $4.0 \pm 3.1$ & $4.2 \pm 3.2$ & $4.7 \pm 3.5$ & $4.7 \pm 3.5$ \\
\hline Depression & $2.9 \pm 2.7$ & $2.9 \pm 2.7$ & $3.3 \pm 2.8$ & $3.1 \pm 2.9$ \\
\hline Physical activity $<1$ h per week & $3140(22.9)$ & $202(17.4)$ & $181(18.2)$ & $179(17.1)$ \\
\hline Education $<10$ years & $2511(18.3)$ & $218(18.7)$ & $181(18.2)$ & $172(16.4)$ \\
\hline Social benefit recipient & $2371(17.3)$ & 189 (16.3) & $241(24.2)$ & $268(25.5)$ \\
\hline Having economic difficulty & $4384(31.9)$ & $274(23.6)$ & 357 (35.8) & $295(28.1)$ \\
\hline Family history of asthma & $2254(16.4)$ & $192(16.5)$ & 188 (18.9) & $190(18.1)$ \\
\hline Alcohol consumption $\geqslant 5$ times per month & $1865(13.6)$ & $182(15.7)$ & $126(12.6)$ & $152(14.5)$ \\
\hline
\end{tabular}

Data are presented as $\mathrm{n}(\%)$ or mean \pm SD. HADS: Hospital Anxiety and Depression Scale. ${ }^{\#}$ : nonusers or those with a daily intake of cod liver oil or multivitamin supplements for $<3$ months.

\section{Results}

Subjects who had complete information on use of cod liver oil and multivitamin supplements were included in the study analysis $(\mathrm{n}=16952)$, and they seemed to have a healthier lifestyle and a better socioeconomic status than those who had missing data on use of cod liver oil or multivitamins $(\mathrm{n}=6614)$ (table 2). In addition, subjects who were included had a higher proportion of family histories of asthma than the excluded subjects.

Among the included subjects, 7\% used cod liver oil only, 6\% used multivitamin supplements only and 6\% used both supplements (table 1). The distributions of age, BMI, education, family history of asthma and alcohol consumption did not show major group differences. Subjects who used both supplements or used multivitamin supplements only were more likely to be female, report receiving social benefits and have chronic disease, and had higher HADS scores of anxiety and depression at baseline than those who used cod liver oil only or were in the reference group.

In comparison with subjects in the reference group, those who used cod liver oil only showed a significantly increased odds ratio for incident asthma (OR 1.59, 95\% CI 1.16-2.19) after adjustment for potential confounding factors including age, sex, smoking, light physical activity, socioeconomic status, family history of asthma and BMI (model I in table 3). Subjects who used multivitamin supplements only also had a significantly increased odds ratio for asthma after the 11-year follow-up compared to the

TABLE 2 Subjects missing information on the use of cod liver oil or multivitamin supplements in in the Nord-Trøndelag Health Study, in comparison with subjects with complete data who were included in the analysis

Subjects with complete data

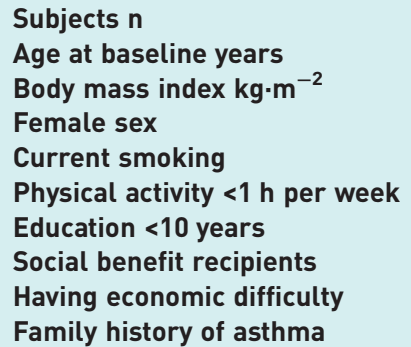

\section{2}

$39.4 \pm 8.8$

$25.6 \pm 3.7$

$9421(55.6)$

4640 (28.9)

$3702(24.2)$

$3082(18.3)$

3069 (19.3)

$5310(31.7)$

$2824(17.7)$
Subjects with missing data

Data are presented as mean \pm SD or $n(\%)$, unless otherwise stated. 
TABLE 3 The association of use of cod liver oil and multivitamin supplements with cumulative incidence of asthma over the 11-year follow-up period of the Nord-Trøndelag Health Study from 1995-1997 to 2006-2008 (N=16952)

\begin{tabular}{|c|c|c|c|c|c|c|}
\hline \multirow{2}{*}{$\begin{array}{l}\text { Regular use of cod liver oil and multivitamin } \\
\text { supplements in the previous year }\end{array}$} & \multirow{2}{*}{$\begin{array}{c}\text { Subjects } \\
\mathrm{n}\end{array}$} & \multirow{2}{*}{$\begin{array}{c}\text { Cases n } \\
\qquad(\%)\end{array}$} & \multirow{2}{*}{$\begin{array}{l}\text { Crude OR } \\
(95 \% \mathrm{CI})\end{array}$} & \multicolumn{3}{|c|}{ Adjusted OR (95\% CI) } \\
\hline & & & & Model I ${ }^{\#}$ & Model II ๆ & Model III ${ }^{+}$ \\
\hline Reference group $\S$ & 13742 & $389(2.8)$ & 1.00 & 1.00 & 1.00 & 1.00 \\
\hline Cod liver oil only & 1163 & $46(4.0)$ & $1.41(1.04-1.93)$ & $1.59(1.16-2.19)$ & $1.59(1.16-2.18)$ & $1.53(1.11-2.11)$ \\
\hline Multivitamin supplements only & 997 & $46(4.6)$ & $1.66(1.21-2.27)$ & $1.55(1.12-2.13)$ & $1.54(1.12-2.13)$ & $1.48(1.07-2.05)$ \\
\hline Use of both & 1050 & $48(4.6)$ & $1.64(1.21-2.23)$ & $1.73(1.26-2.37)$ & $1.73(1.26-2.36)$ & $1.65(1.20-2.26)$ \\
\hline
\end{tabular}

\#: adjusted for age, sex, smoking (current, former, never and unknown), light physical activity, education, social benefit, economic difficulties, family history of asthma and body mass index; ${ }^{\uparrow}$ : adjusted for alcohol consumption in addition to the covariates in model I; ${ }^{+}$: adjusted for Hospital Anxiety and Depression Scale (HADS) scores for anxiety and depression, and chronic disease at baseline, in addition to the covariates in model I ( $n=16819$ due to missing HADS scores); ${ }^{\S}$ : nonusers or those with daily intake of cod liver oil or multivitamin supplements for $<3$ months.

reference group (OR 1.55, 95\% CI 1.12-2.13). The odds ratio for incident asthma in association with use of both supplements was similar to the odds ratios associated with cod liver oil only or multivitamin supplements only (OR 1.73, 95\% CI 1.26-2.37). The pattern of associations remained after stratification by sex, age, smoking, obesity or family history of asthma (data not presented). Additional adjustment for alcohol consumption (model II), or symptoms of anxiety and depression, and chronic disease (model III), showed similar results. Further adjustment for physician visits during the past year and hospitalisation during the past 5 years before baseline after model III did not show major change in the finding (OR 1.57, 95\% CI 1.11-2.21).

In sensitivity analysis when asthma was defined by a stricter definition, the associations remained similar (table 4). The odds ratios for cod liver oil only, multivitamin supplements only and both were 1.72, 1.50 and 1.90, respectively, after adjustment for covariates included in model I. The mean or median duration of intake of multivitamin supplements was $\sim 8$ months among the users. There seemed to be a dose-response relationship between the duration of multivitamin supplements use and risk of incident asthma: above the 3-month cut-point, each additional month of intake of multivitamin supplements was associated with an odds ratio of 1.03 (95\% CI 1.00-1.06) for incident asthma after adjustment for the covariates in model I and additional adjustment for cod liver oil intake for $\geqslant 3$ months.

After exclusion of subjects who reported ever having had COPD, chronic bronchitis or emphysema in HUNT3, similar odds ratios were obtained for use of cod liver oil only, multivitamin supplements only or both after adjustment of covariates in model I (1.55, 1.62 and 1.61, respectively) (online supplementary table 1). A comparable pattern was also shown in a younger group (age $<40$ years at baseline, supplementary table 2 ) and the results were consistent when participants with chronic disease at baseline were excluded (supplementary table 3).

TABLE 4 Sensitivity analysis using a stricter definition for asthma ${ }^{\#}(\mathrm{~N}=14939)$

\begin{tabular}{|c|c|c|c|c|c|c|}
\hline \multirow{2}{*}{$\begin{array}{l}\text { Regular use of cod liver oil and multivitamin } \\
\text { supplements in the previous year }\end{array}$} & \multirow{2}{*}{$\begin{array}{l}\text { Subjects } \\
\mathrm{n}\end{array}$} & \multirow{2}{*}{$\begin{array}{l}\text { Cases n } \\
(\%)\end{array}$} & \multirow{2}{*}{$\begin{array}{l}\text { Crude OR } \\
(95 \% \mathrm{CI})\end{array}$} & \multicolumn{3}{|c|}{ Adjusted OR $(95 \% \mathrm{CI})$} \\
\hline & & & & Model I" & Model II ${ }^{+}$ & Model III ${ }^{\S}$ \\
\hline Cod liver oil only & 1008 & $29(2.9)$ & $1.49(1.00-2.20)$ & $1.72(1.16-2.56)$ & $1.71(1.15-2.54)$ & $1.63(1.09-2.44)$ \\
\hline Multivitamin supplements only & 861 & $26(3.0)$ & $1.56(1.04-2.36)$ & $1.50(0.99-2.28)$ & $1.49(0.98-2.27)$ & $1.43(0.94-2.18)$ \\
\hline Use of both & 895 & $30(3.4)$ & $1.74(1.18-2.56)$ & $1.90(1.28-2.83)$ & $1.90(1.28-2.83)$ & $1.81(1.21-2.70)$ \\
\hline
\end{tabular}

The association of the use of cod liver oil and multivitamin supplements with cumulative incidence of asthma over the 11-year follow-up period of the Nord-Trøndelag Health Study from 1995-1997 to 2006-2008. \#: stricter definition for asthma was participants who were free from wheeze and asthma at baseline who reported asthma at follow-up in combination with use of asthma medication; participants who were free from wheeze and asthma both at baseline and at follow-up served as reference group. ๆ: adjusted for age, sex, smoking lcurrent, former, never and unknown), light physical activity, education, social benefit, economic difficulties, family history of asthma and body mass index. ${ }^{+}$: adjusted for alcohol consumption in addition to the covariates in model I. §: adjusted for Hospital Anxiety and Depression Scale (HADS) scores for anxiety and depression, and chronic disease at baseline, in addition to the covariates in model I ( $\mathrm{n}=14827$ due to missing HADS scores). ${ }^{f}$ : nonusers or those with daily intake of cod liver oil or multivitamin supplements for $<3$ months. 


\section{Discussion}

In this cohort of 16952 participants, we found that users of multivitamin supplements only had an increased odds ratio for incident asthma after the 11-year follow-up; use of cod liver oil only or use of both supplements demonstrated similar odds ratios to that of the multivitamin supplement-only users after adjustment for a complete panel of possible confounding factors.

Our observation on use of cod liver oil only in the current study confirmed our previous finding of a significantly increased risk of asthma in those who used a previous formula of cod liver oil containing a high dose of vitamin A [10]. Two previous studies have investigated the association of multivitamin supplements use with asthma among children [5, 6]. No association was found in a Swedish cross-sectional study [5], while in a prospective study from the USA, use of multivitamin supplements during the first 6 months of life was associated with an increased risk of asthma after 3 years follow-up [6]. Our study is novel and extends previous findings by investigating an adult population with a longer follow-up time (11 years).

The high concentration of vitamin A in the previous formula of cod liver oil was suggested as the main reason for the increased risk of asthma among Norwegian adults using cod liver oil in our previous study [10]. The mechanisms for an increased odds ratio of incident asthma by use of multivitamin supplements are unclear. However, the similar odds ratio for use of both supplements as the use of individual supplement suggests a common pathway. Otherwise, the odds ratio for use of both supplements would have at least been additive. A high concentration of vitamin A in the lung may produce an acute, localised form of intoxication, which is recognised as "asthma" [16]. Unlike the former formula cod liver oil, which contained a high dose of vitamin $\mathrm{A}$ in an oil-based form, multivitamin supplements contained water-miscible or solid forms of vitamin $\mathrm{A}$ in the normal range according to the Norwegian daily recommended need [8]. However, studies suggest that water-miscible or solid forms of vitamin A preparations are about 10 times as toxic as oil-based vitamin A preparations [17]. It has also been reported that the adult Norwegian population has a relatively high dietary intake of vitamin A [9, 18]. Together with a diet already rich in vitamin $\mathrm{A}$, the vitamin $\mathrm{A}$ in the multivitamin supplements may have contributed to the increased risk of adult asthma in our study.

Apart from vitamin A, vitamins $\mathrm{D}$ and $\mathrm{E}$ are also common components in both cod liver oil and multivitamin supplements. However, current large population studies showed either no association [19, 20] or a protective association of higher serum 25-hydroxyvitamin D levels with incident asthma [21-23]. With respect to the effect of vitamin $\mathrm{E}$ on incident asthma, the majority of scientific evidence does not show any detrimental effect [24-26]. Therefore, both vitamins D and E are less likely to explain our findings. In addition to the hypothesis on vitamin A, both cod liver oil and multivitamin supplements are combinational formula of different vitamins; it is possible that the interactions of vitamins have played a role in asthma risk.

Scientific evidence regarding the beneficial or harmful effect of multivitamin supplements in relation to asthma is scarce. To our knowledge, the current study is one of the few prospective studies evaluating the association of multivitamin supplement intake with onset of asthma in adults over a long follow-up period. Moreover, the study population is considerably large and relatively homogeneous. In this study, we also had the possibility to adjust for many potential confounders, such as lifestyle factors and chronic diseases.

However, our study may have several potential limitations. First, selection bias might exist since the included population seemed to be different from the population with missing data on cod liver oil or multivitamin supplements use with regard to social economic status and lifestyle factors. However, these factors are more likely to be confounding factors than consequences of the supplement use and asthma outcome, and therefore more appropriate to adjust for. Selection bias, if any, would have resulted in conservative estimate for our findings since the included people seemed to have a higher social economic status but multivitamin supplements use was associated with a lower social economic status.

Furthermore, some confounders, such as unhealthy lifestyles, comorbidities and diet, might also contribute to our findings. Unfortunately, we do not have data on diet, but no single diet has been confirmed to be associated with asthma risk. In addition, social-economic status can be a proxy measure for diet as it is closely related to diet [27]. When we adjusted for various unhealthy lifestyle factors and chronic diseases, there were no substantial changes in the results. Restricting the analysis in subjects with no chronic disease or age $<40$ years (a heathier group), we observed similar results. However, it is worth noting that people who used multivitamin supplements were more likely to report having chronic diseases than nonusers and, therefore, asthma may be more likely to be diagnosed in the multivitamin supplement users due to frequent consultations of a physician for other diseases. This may partially explain the positive association between multivitamin use and incident asthma we observed but further adjustment of physician visits during the past year and hospitalisation during the past 5 years before baseline after model III cannot change our conclusion. 
Information bias might also influence our results. Intake of cod liver oil or multivitamin supplements was assessed by self-administered questionnaire, which might lead to some attenuation of the association due to nondifferential misclassification. We were also unable to carefully investigate the dose-response relationship due to lack of information on the dosage of vitamins. However, there seemed to be a dose-response association between months of multivitamin use and asthma risk. Additionally, in the original HUNT2 questionnaire, the words "vitamin supplements" rather than "multivitamin supplements" were used. Although most of the people were assumed to take multivitamin supplements, single use of vitamin B, C and D could not be excluded. Vitamins B and C are not contained in the cod liver oil product, and therefore, are less likely to explain the common pathway for multivitamin and cod liver oil supplements. Vitamin D has been discussed in the previous section to have either a protective or no association with asthma risk [19-23]. Finally, there is no established cut-point to define regular intake of multivitamin supplements. We used $\geqslant 3$ months' use of multivitamin supplements during the previous year to reflect regular intake. Despite being a somewhat arbitrary choice of cut-point, we found similar results as using either $\geqslant 1$ month or $\geqslant 8$ months as the cut-point (data not presented). The 8 -month cut-point was the mean duration of intake among users but showed reduced power in subgroup analysis.

There was also a possible misclassification of asthma caused by self-reporting. Many studies, however, have reported that the validity and reliability of self-reported asthma are acceptable $[28,29]$. In the present study, we did a sensitivity analysis by using a stricter asthma definition (confirmation of asthma medicine usage combined with self-reported asthma) and further excluded subjects who reported ever having had COPD, chronic bronchitis or emphysema in the data analysis. We did not find any notable differences in results.

Overall, we found that intake of multivitamin supplements was associated with an increased odds ratio for incidence of asthma in Norwegian adults after an 11-year follow-up. Confirmation of this observation and possible mechanisms driving this association deserve further investigation.

\section{Acknowledgements}

The Nord-Trøndelag Health Study (The HUNT Study) is collaboration between HUNT Research Centre (Faculty of Medicine, Norwegian University of Science and Technology (NTNU)), Nord-Trøndelag County Council and the Norwegian Institute of Public Health.

\section{References}

1 Comerford KB. Recent developments in multivitamin/mineral research. Adv Nutr 2013; 4: 644-656.

2 Kamangar F, Emadi A. Vitamin and mineral supplements: do we really need them? Int J Prev Med 2012; 3: 221.

3 Larsson SC, Åkesson A, Bergkvist L, et al. Multivitamin use and breast cancer incidence in a prospective cohort of Swedish women. Am J Clin Nutr 2010; 91: 1268-1272.

4 Zhang Y, Coogan P, Palmer JR, et al. Vitamin and mineral use and risk of prostate cancer: the case-control surveillance study. Cancer Causes Control 2009; 20: 691-698.

5 Marmsjö K, Rosenlund H, Kull I, et al. Use of multivitamin supplements in relation to allergic disease in 8-y-old children. Am J Clin Nutr 2009; 90: 1693-1698.

6 Milner JD, Stein DM, McCarter R, et al. Early infant multivitamin supplementation is associated with increased risk for food allergy and asthma. Pediatrics 2004; 114: 27-32.

7 Danielsen S, Ekrol PS. Food supplements in the Nordic countries - results from surveys among consumers in the Nordic countries. Oslo, Mattilsynet, 2009.

8 Blomhoff RB-SU, Brot C, Solvoll K, et al. Health risks related to high intake of preformed retinol (vitamin A) in the Nordic countries. Copenhagen, Nordic Council of Minsters, 2003.

9 Forsmo S, Fjeldbo SK, Langhammer A. Childhood cod liver oil consumption and bone mineral density in a population-based cohort of peri-and postmenopausal women: the Nord-Trøndelag Health Study. Am J Epidemiol 2008; 167: 406-411.

10 Mai X-M, Langhammer A, Chen Y, et al. Cod liver oil intake and incidence of asthma in Norwegian adults - the HUNT study. Thorax 2013; 68: 25-30.

11 Holmen J, Midthjell K, Krüger Ø, et al. The Nord-Trøndelag Health Study 1995-97 (HUNT 2): objectives, contents, methods and participation. Norsk Epidemiol 2003; 13: 19-32.

12 Krokstad S, Langhammer A, Hveem K, et al. Cohort profile: the HUNT study, Norway. Int J Epidemiol 2013; 42: 968-977.

13 World Health Organization. Preventing and managing the global epidemic: report of a WHO consultation on obesity. Geneva, World Health Organization, 2004.

14 Snaith RP. The hospital anxiety and depression scale. Health Qual Life Outcomes 2003; 1: 29.

15 Bjelland I, Dahl AA, Haug TT, et al. The validity of the Hospital Anxiety and Depression Scale: an updated literature review. J Psychosom Res 2002; 52: 69-77.

16 Mawson A. Could bronchial asthma be an endogenous, pulmonary expression of retinoid intoxication? Front Biosci 2001; 6: D973-D985.

17 Myhre AM, Carlsen MH, Bohn SK, et al. Water-miscible, emulsified, and solid forms of retinol supplements are more toxic than oil-based preparations. Am J Clin Nutr 2003; 78: 1152-1159.

18 Johansen AMW, Lie RT, Wilcox AJ, et al. Maternal dietary intake of vitamin A and risk of orofacial clefts: a population-based case-control study in Norway. Am J Epidemiol 2008; 167: 1164-1170.

19 Confino-Cohen R, Brufman I, Goldberg A, et al. Vitamin D, asthma prevalence and asthma exacerbations: a large adult population-based study. Allergy 2014; 69: 1673-1680. 
20 Cheng HM, Kim S, Park GH, et al. Low vitamin D levels are associated with atopic dermatitis, but not allergic rhinitis, asthma, or IgE sensitization, in the adult Korean population. J Allergy Clin Immunol 2014; 133: $1048-1055$.

21 Mai X-M, Langhammer A, Camargo CA, et al. Serum 25-hydroxyvitamin D levels and incident asthma in adults: the HUNT study. Am J Epidemiol 2012; 235.

22 Frieri M, Valluri A. Vitamin D deficiency as a risk factor for allergic disorders and immune mechanisms. Allergy Asthma Proc 2011; 32: 438-444.

23 Bozzetto S, Carraro S, Giordano G, et al. Asthma, allergy and respiratory infections: the vitamin D hypothesis. Allergy 2012; 67: 10-17.

24 Allen S, Britton J, Leonardi-Bee J. Association between antioxidant vitamins and asthma outcome measures: systematic review and meta-analysis. Thorax 2009; 64: 610-619.

25 Troisi RJ, Willett WC, Weiss ST, et al. A prospective study of diet and adult-onset asthma. Am J Respir Crit Care Med 1995; 151: 1401-1408.

26 Devereux G, Turner SW, Craig LC, et al. Low maternal vitamin E intake during pregnancy is associated with asthma in 5-year-old children. Am J Respir Crit Care Med 2006; 174: 499-507.

27 Darmon N, Drewnowski A. Does social class predict diet quality? Am J Clin Nutr 2008; 87: 1107-1117.

28 De Marco R, Cerveri I, Bugiani M, et al. An undetected burden of asthma in Italy: the relationship between clinical and epidemiological diagnosis of asthma. Eur Respir J 1998; 11: 599-605.

29 Brisman J. Asthma and asthma-like symptoms in adults assessed by questionnaires. A literature review. Chest 1993; 104: 600-608. 